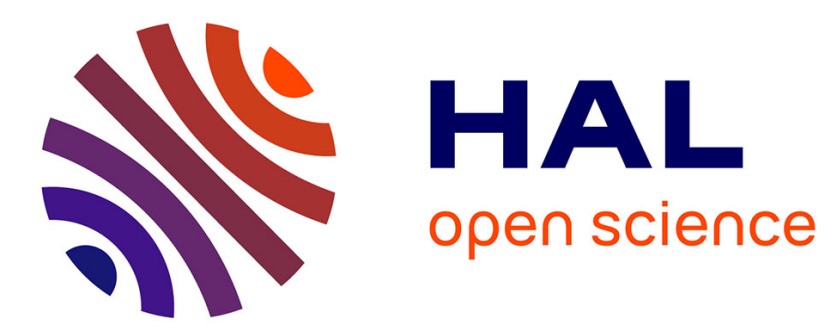

\title{
À tout âge, le bonheur de vieillir !
}

Gérard-François Dumont

\section{To cite this version:}

Gérard-François Dumont. À tout âge, le bonheur de vieillir!. Population et avenir, 2009, 695, pp.3. 10.3917/popav.695.0003 . halshs-00764908

\section{HAL Id: halshs-00764908 https://shs.hal.science/halshs-00764908}

Submitted on 13 Dec 2012

HAL is a multi-disciplinary open access archive for the deposit and dissemination of scientific research documents, whether they are published or not. The documents may come from teaching and research institutions in France or abroad, or from public or private research centers.
L'archive ouverte pluridisciplinaire HAL, est destinée au dépôt et à la diffusion de documents scientifiques de niveau recherche, publiés ou non, émanant des établissements d'enseignement et de recherche français ou étrangers, des laboratoires publics ou privés. 


\section{À tout âge, le bonheur de vieillir !}

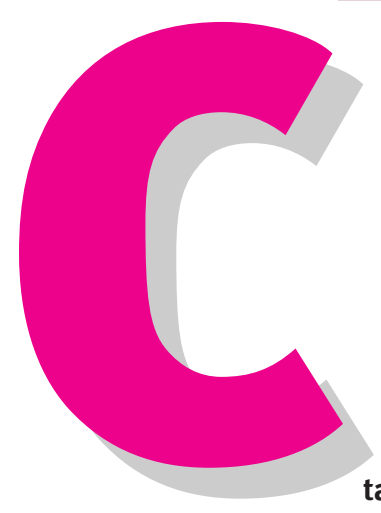

par Gérard-François DUMONT

omme le temps passe ! Le $\mathrm{XXI}^{\mathrm{e}}$ siècle finit sa première décennie. Il prépare ces années 2010 dont chacun espère la fin de la crise et la grande mutation du monde vers la paix sur tous ses territoires, une bonne gouvernance et un développement durable ${ }^{1}$. Faut-il avoir la nostalgie des années, des mois et des jours qui s'accumulent derrière nous ?

Doit-on penser que cette nostalgie devrait être encore plus intense pour les plus âgés d'entre nous ? Le général de Gaulle n'a-t-il pas effectivement écrit : « La vieillesse est un naufrage ${ }^{2}$ ? Mais il écrivait cette formule à propos du Maréchal Pétain en 1940. Pourtant, depuis cette date, l'espérance de vie à la naissance s'est accrue en France d'un quart de siècle, de 56 ans à plus de 81 ans, une évolution que personne n'avait imaginée. Ainsi se constate un décalage fantastique entre les progrès dans la longévité accrue et les propos apocalyptiques souvent déversés dans les médias. Travaillons à un monde meilleur, mais ne pensons pas que les générations qui nous ont précédés n'ont rien fait en matière d'environnement ${ }^{3}$. En effet, les gains d'espérance de vie résultent d'un ensemble de facteurs combinant une alimentation suffisante et plus diversifiée, des découvertes médicales et pharmaceutiques, des progrès techniques limitant la pénibilité du travail et de considérables changements touchant l'hygiène. Ces derniers additionnent les meilleurs équipements des logements en sanitaires et salles d'eau ou des multiples infrastructures urbaines contribuant à combattre morbidité et mortalité : réseaux d'eau potable, assainissement, traitement des eaux usées, stations d'épuration...

La science démographique répond clairement à la question ci-dessus.

D'une part, elle confirme une évidence : vieillir est la meilleure façon de ne pas mourir. Le vieillissement individuel $^{4}$ est donc une excellente solution pour continuer à bénéficier de la vie.

D'autre part, non seulement celui qui vieillit ne meurt pas mais, en outre, dans un pays comme la France, il allonge son espérance totale de vie. La connaissance des évolutions démographiques apprend que plus une personne avance en âge, plus elle peut espérer une vie plus longue. Par exemple, en 2006, l'espérance de vie à la naissance en France est de 81 ans en moyenne, davantage pour les femmes et moins pour les hommes. Mais la personne qui a atteint l'âge de 85 ans dispose encore, en moyenne, d'une espérance de vie de 5,96 années pour les hommes et de 7,40 années pour les femmes.

La capacité d'allonger son espérance totale de vie en vieillissant peut être mise en évidence en France en considérant
LA DURÉE MOYENNE DE VIE ESCOMPTÉE d'UNE PERSONNE NÉE EN 1920 En FRANCE MÉTROPOLITAINE SELON SON ÂGE ATTEINT

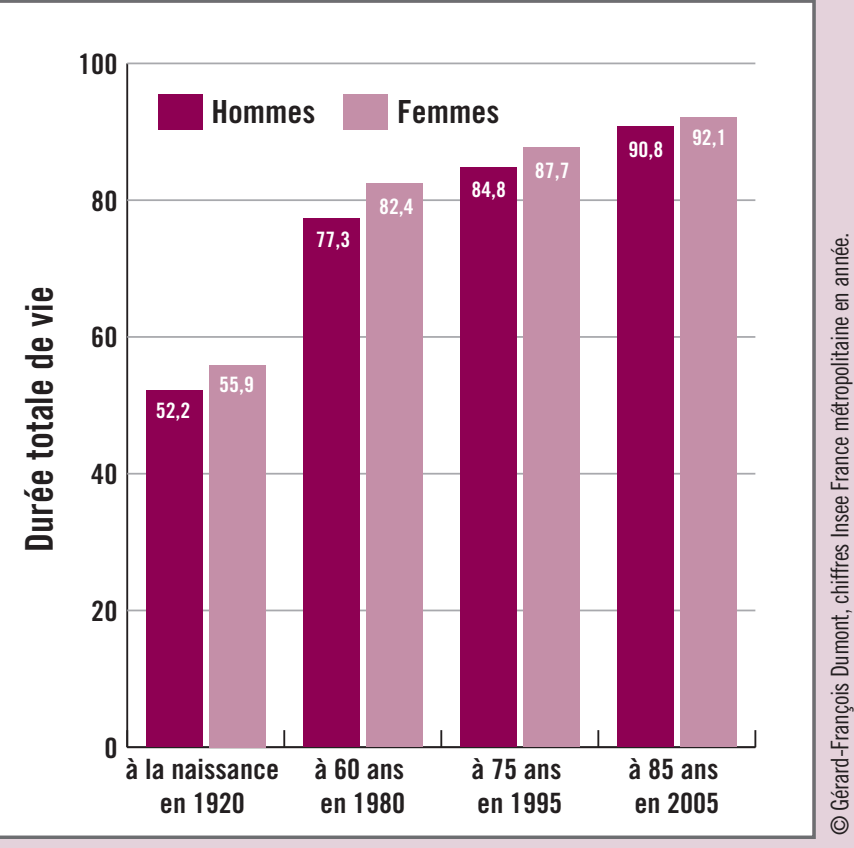

par exemple la génération née en 1920. Les nouveau-nés de cette année-là ont des espoirs limités de vivre. Déjà, passer le cap de la première année sera difficile. 123 sur mille vont mourir avant même d'atteindre l'âge d'un an. L'espérance de vie à la naissance de la génération 1920 est seulement de 55,9 ans pour les femmes et de 52,2 pour les hommes. Mais ceux qui, au fil des années, survivent montrent une meilleure résistance aux causes naturelles de la mortalité et bénéficient des facteurs qui améliorent l'espérance de vie, comme la découverte des antibiotiques. Aussi, ceux arrivant à l'âge de 60 ans en 1980 comptent une espérance de vie restante de 22,4 ans pour les femmes et de 17,3 ans pour les hommes. Leur durée espérée de vie atteint donc respectivement 82,4 ans et 77,3 ans, soit beaucoup plus qu'à leur naissance en 1920.

Quinze ans plus tard, les personnes de cette même génération nées en 1920 qui atteignent 75 ans en 1995 ont, devant elles, une durée espérée de vie encore plus longue : 87,7 ans pour les femmes et 84,8 pour les hommes. Là aussi, ces personnes bénéficient des conséquences des nouveaux progrès réalisés, notamment dans la médecine gérontologue. Considérons désormais les survivants de la génération 1920 arrivés à l'âge de 85 ans en 2005 : leur durée espérée de vie s'élève désormais à 92,1 ans pour les femmes et 90,8 ans pour les hommes.

De tels chiffres illustrent tout l'intérêt de vieillir pour allonger sa durée espérée de vie. Ils permettent aussi de mieux éclairer la réflexion sur les politiques de l'État et des collectivités territoriales.
1. Concernant les États-Unis, cf. Zaninetti, Jean-Marc, «Les États-Unis face au développement durable », Population \& Avenir, no 690, now Avenir, $n^{\circ} 690$, novembredécembre 2008.

2. Charles de Gaulle, Mémoires de guerre : L'Appel, 1940-1942, Livre de Poche.

3. Wackermann, Gabriel (Direction), Ville et environnement, Paris, Ellipses, 2005.

4. Notion à bien distinguer $\mathrm{du}$ vieillissement de la population. Cf. Dumont, Gérard-François et alii, Les territoires face au vieillissement en France et en Europe, Paris, Ellipses, 2006. 